RUNNING HEAD: Bursting with anxiety

\title{
Bursting with Anxiety: Adult Social Referencing in an Interpersonal Balloon Analogue Risk Task (BART)
}

\author{
Brian Parkinson, Natasha Phiri, and Gwenda Simons \\ Department of Experimental Psychology \\ University of Oxford, UK
}

Address correspondence to:

\author{
Brian Parkinson \\ Department of Experimental Psychology \\ University of Oxford \\ South Parks Road \\ Oxford OX1 3UD \\ UK
}

Keywords: affect, facial expression, risk, social appraisal 


\begin{abstract}
This study developed an interpersonal modification of the Balloon Analogue Risk Task (BART) to assess effects of a friend's real-time facial expressions on co-participants' risk-taking. Twenty pairs of male friends and 20 pairs of female friends completed two counterbalanced versions of BART, one framed in terms of monetary gain, and the other framed in terms of avoiding loss. Each pair included a player who performed the task across both trials and a reference person in a separate cubicle connected via a soundless video link. In the suppression condition, reference persons were told to minimise facial expressions of anxiety as balloons inflated. In the expression condition, they were told to freely express anxiety. As predicted, players took greater risks and burst more balloons in the suppression condition, but only under the gain frame. Players' BART scores across both frames were also significantly negatively correlated with reference persons' scores on a questionnaire measure of dispositional expressivity (BEQ), confirming that other people's expressions can moderate own risk-taking.
\end{abstract}

Key words: Social referencing, anxiety, facial expression, emotion suppression, framing, risk 


\section{Bursting with Anxiety: Adult Social Referencing in an Interpersonal Balloon Analogue Risk Task (BART)}

Effects of emotions on decision-making are well-documented (e.g., Loewenstein, Weber, Hsee, \& Welch, 2001). For example, fear and anxiety often increase sensitivity to threat, and lead to more risk-averse decisions (e.g., Lerner \& Keltner, 2001). Less research attention has been given to possible effects of other people's emotions in interpersonal situations (Parkinson, 1996; van Kleef, 2009). In particular, does someone else's apparent anxiety also influence threat sensitivity and risk-taking? Is a person's orientation to uncertain situations influenced by the perceived emotional orientation of others?

\section{Social referencing and social appraisal}

Social referencing studies by developmental psychologists provide clear examples of this kind of interpersonal effect. For instance, Sorce and colleagues showed that one-year-old infants were more likely to cross a visual cliff when their mothers smiled at them from the other side than when mothers posed fear faces (Sorce et al., 1985). Thus, a close other's emotion expressions influenced children's orientation towards uncertain situations, clarifying their evaluation. The usual interpretation of these findings is that toddlers base their appraisals of the potential threat on information derived from observed expressions (social appraisal in Manstead \& Fischer's 2001 terms).

Social referencing is often seen as part of a developmental stage that children pass through before learning to internalize symbolic meanings of facial expressions and more sophisticated strategies for regulating their own emotions (e.g., Holodynski \& Friedlmeier, 2006). If so, interpersonal processes of social referencing may be superseded by intrapersonal 
self-regulation as children get older. However, it seems possible that some forms of social referencing also characterise many adult-adult interactions, especially when the emotional meaning of the situation is ambiguous. For example, participants in Latané and Darley's (1968) famous study of reactions to a simulated emergency were slower to respond to artificial smoke when they waited with unresponsive confederates than when they waited either alone or with other naïve participants. In other words, the apparent calmness displayed by others may have signalled to participants that the situation was not as risky as they might otherwise have suspected. Correspondingly, other adults' anxiety might indicate higher levels of risk.

Parkinson and Simons (2009) collected diary data supporting this prediction. Participants' anxiety about impending everyday decisions was significantly affected by the reported anxiety of another person with whom they interacted, and this interpersonal affect transfer was partly mediated by appraisals of risk. In other words, if someone else is evidently worried about potential outcomes of a planned course of action, that can make these outcomes seem riskier and consequently increase one's own level of anxiety.

Because Parkinson and Simons' (2009) data were collected in ecologically valid settings, a number of interrelated processes may have contributed to reported effects. First, third variables such as extraneous risk cues may have exerted parallel effects on others' and own anxiety rather than others' anxiety directly influencing own anxiety. Although the researchers attempted to control for such effects statistically, unmeasured or uncontrolled factors may still have played some role. An experimental manipulation of others' anxiety expression should permit more definitive conclusions about causation.

Second, anxiety may have been communicated from person to person by a variety of channels in Parkinson and Simons' (2009) study. In particular, facially expressed information 
was probably supplemented by verbal communication of worries. Removing speech from interpersonal interaction seems necessary to establish nonverbal influence more clearly.

Finally, for practical reasons, Parkinson and Simons' (2009) diary procedure did not include any behavioral measures of decision-making. Thus, the results provided no direct evidence that the reported effects on risk-related judgements and appraisals were translated into action. To address this limitation, the present study incorporated a performance-based measure of risk-taking based on the Balloon Analogue Risk Task (Lejuez et al., 2002).

\section{Balloon Analogue Risk Task (BART)}

BART is a computer game in which participants' key-presses progressively inflate the image of a balloon on a monitor. On each trial, more points are gained as the balloon grows larger, but over-inflation bursts the balloon leading to the loss of points from the temporary bank for that trial. Balloons are programmed to pop after different numbers of key-presses, making the outcome of each trial unpredictable. BART has become one of the most widely used performance measures of risk behavior (Harrison, Young, Butow, Salkeld, \& Solomon, 2005), and has been shown to relate to real-world risk-taking (e.g. smoking, drug use, unprotected sex) across a broad range of studies (e.g., Aklin et al, 2005, Bornovalova et al, 2008, Crowley et al, 2006, Lejuez et al., 2002). Although BART is most commonly seen as a trait measure of risktaking propensity, a number of studies have also adapted it for assessment of temporary states of risk preference (e.g., Benjamin \& Robbins, 2007; Heilman et al., 2010). As well as providing an objective index of risky choices, its main advantage for the present research is its sequential nature, which permits manipulation of real-time expressive feedback from another person.

This study adapted BART for use as a game for pairs of participants, one of whom took 
the role of reference person (i.e., observed the task and provided nonverbal feedback) while the player pressed the key to inflate the balloon. Communication between co-participants was restricted to the facial channel by situating them in different cubicles where their only contact was via a soundless video link. Reference persons' emotion expressions were manipulated by separately instructing them either to freely express or to suppress facial indications of anxiety. Suppression of expression is a commonly deployed emotion-regulation strategy (see Gross, 1998) that is often used when interacting with close others (e.g., Butler et al., 2003; Butler \& Gross, 2004), so we expected our participants to be well-practised in achieving the instructed outcome (see also Lanzetta, Cartwright-Smith, \& Kleck, 1976). Our primary hypothesis based on research into social referencing (e.g., Sorce et al., 1985) and social appraisal (e.g., Parkinson \& Simons, 2009) was that participants would be discouraged from risk-taking by reference persons' increased anxiety expressions leading to lower BART scores (i.e. less risk-taking) in the expression than in the suppression condition. Appraisal information conveyed by the reference person's anxiety expression should similarly lead to increased risk appraisal and experienced anxiety in the player.

Interpersonal effects of reference persons' anxiety expressions should also depend on their characteristic level of facial expressivity. Reference persons who are dispositionally more expressive should produce stronger and more readable anxiety expressions, which in turn should discourage players from risk-taking to a greater extent. We therefore predicted lower BART scores for players whose reference persons were relatively more expressive. Assuming that the reduction in nonverbal signals of anxiety is neither complete nor constant under suppression instructions (e.g., Ekman \& Friesen, 1969), this effect should apply across both expression conditions. We measured dispositional expressivity using a widely used and validated 
questionnaire measure, the Berkeley Expressivity Questionnaire (BEQ, Gross \& John, 1995).

\section{Framing}

A second aim of the present study was to investigate effects of framing on risk behavior in an interpersonal context, and the possible moderation of these effects by reference persons' anxiety expressions. According to prospect theory (Kahneman \& Tversky, 1979), individuals become less risk-averse when decision options are framed in terms of losses rather than gains, because of the relatively greater subjective value attached to loss avoidance. Several studies have supported this prediction (e.g., Brewer \& Kramer, 1986), although the consistency of findings varies across different kinds of task and framing manipulation (Levin, Schneider, \& Gaeth, 1998). Krishnamurthy and Kumar (2002, study 3) found that framing effects were smaller for judgements about another person's risk preference rather than one's own. One possible explanation is that framing's influence depends on affective states induced by orientations to loss or gain (e.g., Fagley, Coleman, \& Simon, 2010), and that the limits of empathy reduce the impact of these states when thinking about someone else's risk preference (cf. Hsee \& Weber, 1997).

Benjamin and Robbins (2007) compared performance on the standard BART where keypresses explicitly produce gains until the balloon bursts (GBART) with performance on a lossframe version of the task (LBART) where key-presses are presented as reducing the amount of losses on each trial. As predicted, risk-taking (as indexed by balloon-pumps) was significantly lower for GBART than LBART. Contrary to predictions based on Krishnamurthy and Kumar's (2002) findings, this framing effect was no weaker when participants completed the BART on behalf of their best friend or a local charity rather than themselves. However, Benjamin and 
Robbins acknowledged that the strength of their interpersonal manipulation may have been compromised because participants only earned hypothetical money in all versions of the task.

Our study assessed whether similar framing effects would also apply to our more directly interpersonal modification of BART in which genuine money was shared between coparticipants. Following Benjamin and Robbins, we predicted that a loss frame would encourage greater risk-taking than a gain frame. Further, thinking about the prospect of losing money may attune people to potentially diagnostic information about the risk of loss including information derived from other people's apparent emotions (i.e., social referencing information). For this reason, we predicted that the effects of our expression manipulation would be stronger in the loss-frame condition. Indeed, any encouragement of risky behaviour resulting from a loss frame might be counteracted or even reversed by social-referencing responses to reference persons' apparent anxiety in the expression condition.

\section{Hypotheses}

In sum, we hypothesized that players' risk scores would be higher (and their risk appraisals and anxiety lower) when reference persons suppressed their anxiety expressions, when reference persons were lower in dispositional expressivity as measured by BEQ (Gross \& John, 1995), and when the task was presented in terms of loss rather than gain (Benjamin \& Robbins, 1997). Finally, we predicted interactions between framing and expression manipulations, with stronger effects of anxiety expression under the loss frame. 


\section{Method}

\section{Participants}

Twenty pairs of male friends and 20 pairs of female friends aged between 18 and 31 ( $M$ $=23.40, S D=3.58$ ) participated either for course credit or a $£ 5$ payment (approximately $\$ 8$ ). All pairs also had the chance of winning up to an additional $£ 10$ (approximately \$16) during the computerized task. Data from three pairs were discarded due to technical problems with the camera or computer software. Data from one additional pair were also discarded because (contrary to instruction) the reference person made overt hand signals to the player during the game. Replacement pairs were recruited in these four cases. Approximately three quarters of the sample (78.3\%) were undergraduate or graduate students, and most were Caucasian (68.3\%).

\section{Design}

The study utilized a 2 (anxiety expression: suppression vs. expression) x 2 (frame: gain vs. loss) mixed factorial design with anxiety expression condition as a between-participants factor and reward frame as a within-participants factor. The main dependent measures were risk scores derived from performance on the BART. Other dependent measures related to reference persons' and players' ratings of their own and each others' anxiety expressions, and players' ratings of their own experienced anxiety and appraisal of risk. Because these variables were assessed for both partners in each pair, we added a further repeated measures (within-pairs) factor of participant role (player vs. reference person) when analyzing them.

\section{BART programming}

Both gain and loss versions of BART were modifications of the tasks used by Benjamin 
and Robbins (GBART and LBART, 2007), programmed with E-Prime software. In both cases, the computer screen displayed a picture of a balloon and indications of the number of pumps made on the current trial and of how many points had been won or lost on previous trials (see Figures 1a and 1b). In the gain task (Figure 1a), 2 additional points were earned for each balloon pump (up arrow key-press) that was not followed by the balloon bursting (up to a maximum of 40 points per balloon). If the player pressed the space bar to bank the profits, points gained for that balloon were added to the cumulative total. If the player pressed the up arrow key too many times causing the balloon to explode, no additional points were added to this total.

The reward structure of the loss task (Figure 1b) was identical, but players were given an initial allocation of 40 points per balloon and told that they would avoid losing points from this allocation by inflating the balloon without bursting it. Two additional points were saved for each pump, and banking any saved points that had been collected (by pressing the space bar) meant that they were subtracted from the maximum number of lost points for each balloon (40), and this number (40 minus collected saved points) was added to the displayed cumulative total of lost points at the end of each trial. Bursting the balloon led to the loss of all 40 points for that balloon.

Unlike Benjamin and Robbins (2007) we did not supplement the display of cumulative points total (across trials) with a separate display of the number of gained or lost points for each balloon because this would have meant that participants in the loss-frame condition saw the score for each balloon gradually approach a minimum of zero, perhaps leading to implicit targets about the proportion of points that should be saved on any trial. The absence of a comparable display of maximum points score per balloon in the gain-frame condition would have meant that the frame manipulation was confounded with explicit provision of ongoing information about 
progress towards a specific value. Since our indication of number of pumps made per balloon translated directly into points won under the gain frame, and points lost under the loss frame, any additional display seemed largely redundant in any case.

Because the task had to be completed twice by each participant pair, we also reduced the highest number of pumps that could produce an explosion to 20 , which also brought the advantage of making each round faster-paced (see also Rao, Korczykowski, Pluta, Hoang, \& Detre, 2008, who reduced the maximum number of pumps per trial to 12 , and Gabriel \& Williamson, 2010, who restricted maximum pumping time per balloon to 20 seconds). We also wanted to ensure that players would have sufficient time to register the reference person's reaction before choosing to continue pumping and therefore increased the minimum number of pumps that could produce an explosion to 5. Each condition included 30 trials (as in Lejuez et al., 2003), and so each pair of participants completed 60 trials in all.

\section{Self-report measures}

When co-participants had completed both GBART and LBART, they separately completed questionnaires concerning their reactions and their perceptions of the co-participant's reactions. Two sets of identical questions applied to the gain and loss versions of BART. In each case, participants were specifically asked to focus on the trial where the player had made the most pumps when answering. A 7-point scale was used to rate all items with endpoints labelled "Not at all" (1) and "Very" (7).

Facial expressions. Participants were asked to rate how anxious and how calm their facial expression had been as the specified balloon was inflating, and how anxious the other participant (player or reference person as appropriate) had seemed. Players were also asked to 
rate the extent to which the reference person appeared more anxious than they did themselves.

Anxiety. Players rated how anxious they had felt as the specified balloon was inflating and the extent to which looking at the reference person had made them feel more anxious.

Risk appraisals. Players rated how much they felt they were taking a risk as the balloon inflated and how much they felt that the reference person thought they were taking a risk.

\section{Berkeley Expressivity Questionnaire (BEQ)}

Individual differences in emotional expressivity were assessed using the BEQ, a 16-item questionnaire containing items such as, "Whenever I feel positive emotions, people can easily see exactly what I am feeling" (Gross \& John, 1995). Participants rated the extent to which they agreed with each item on a 7-point scale ranging from strongly disagree (1) to strongly agree (7). Overall scores are based on the mean of three sub-scale scores $(\alpha=.73$, for the present sample).

\section{Equipment}

Two separate cubicles were used for players and reference persons, each containing a networked Dell computer linked to two separate 19-inch monitors, and a wall-mounted video camera (FVS CL3000WDR/MV) positioned behind these monitors. One of the monitors in each cubicle showed a real-time video feed of the co-participant's face. The other showed the ongoing progress of BART as controlled by the player, with the signal transmitted to the reference person's cubicle using a splitter cable. The positioning of the monitors showing BART and the video feed of the co-participant's face were mirrored across cubicles allowing participants to look directly at each other's faces. 


\section{Procedure}

Pairs of friends were told that they would complete two rounds of the "Balloon Puffers" game (Benjamin \& Robbins, 2007), and that they could jointly earn a maximum of 1200 points, worth $£ 5$ (approximately $\$ 8$ ) of real money in each round. One participant in each pair was randomly allocated to the role of player and the other to the role of reference person and these roles were maintained across both rounds with either GBART or LBART first depending on counterbalancing. The experimenter explained the rules of the game and informed participants that they would not be able to converse during the task but would be able to see each other via a video link (ostensibly in order to allow the reference person to make judgements about the player's performance). After seating reference persons in the separate cubicle, the experimenter gave them additional instructions without players' knowledge to permit the manipulation of anxiety expression. Reference persons in the anxiety-expression condition were told to freely express any anxiety they experienced, whereas reference persons in the anxiety-suppression condition were told to suppress any expression of anxiety (photographs of co-participants performing BART in each of these conditions are shown in Figure 2). The experimenter then switched on the video feed and started the first round.

When both rounds were finished, participants completed the questionnaires in their separate cubicles with the video feed turned off. Next, players were asked questions designed to determine if they suspected that reference persons had been suppressing or enhancing facial expressions (but none did). Finally, a full debriefing was provided to all participants. 


\section{Results}

\section{Expression manipulation checks}

Self-ratings of expressed anxiety and calmness were subjected to 2 X 2 X 2 ANOVAs using reward frame, expression manipulation, and participant role as independent variables. Both analyses revealed significant main effects of the expression manipulation (anxiety $F(1,38)$ $=21.03, p<.001 \eta_{p}^{2}=.36$; calmness $\left.F(1,38)=18.97, p<.001 \eta_{p}{ }^{2}=.33\right)$, significant or nearsignificant main effects of participant role (anxiety $F(1,38)=6.17, p=.018, \eta_{p}{ }^{2}=.14$;

calmness $\left.F(1,38)=3.71, p=.062, \eta_{p}{ }^{2}=.09\right)$, and significant two-way interactions between these variables (anxiety $F(1,38)=31.23, p<.001, \eta_{p}{ }^{2}=.45$; calmness $F(1,38)=14.44, p=$ $\left..001, \eta_{p}{ }^{2}=.35\right)$. None of the other effects were significant. Ratings of anxiety expression were higher $(M=4.80, S D=1.05)$, and ratings of calm expressions lower $(M=3.24, S D=.96)$, in the anxiety expression than in the anxiety suppression condition (anxiety: $M=3.35, S D=.94$; calm: $M=4.55, S D=.95)$. Further, players' self-ratings of expressed anxiety were higher $(M=4.38$, $S D=1.27)$, and their self-ratings of expressed calmness were lower $(M=3.63, S D=1.40)$ than the corresponding self-ratings by reference persons (anxiety: $M=3.78, S D=1.87$; calm: $M=$ 4.16, $S D=1.74)$.

Decomposing the two-way interaction, analysis of simple main effects revealed that, as intended, reference persons rated their expressed anxiety as significantly higher, and their expressed calmness as significantly lower in the anxiety-expression condition (anxiety: $M=5.18$, $S D=1.35$; calm: $M=2.88, S D=1.02)$ than in the anxiety-suppression condition (anxiety: $M=$ $2.38, S D=1.10$; calm: $M=5.45, S D=1.29 ;$ anxiety $F(1,38)=51.73, p<.001, \eta_{p}{ }^{2}=.58$; calmness $\left.F(1,38)=49.03, p<.001, \eta_{p}{ }^{2}=.56\right)$, confirming the success of the expression manipulation. By contrast, there were no significant effects of expression condition on players' 
self-rated anxiety or calmness expressions $(F \mathrm{~s}<.05)$.

The same pattern of significant effects was found when analyzing co-participants' perceptions of each other's anxiety expressions in place of self-reports. Again, there were significant main effects of expression condition $\left(F(1,37)=4.87, p=.034, \eta_{p}{ }^{2}=.12\right)$ and participant role $\left(F(1,37)=4.72, p=.036, \eta_{p}{ }^{2}=.11\right)$, and a significant two-way interaction between these variables $\left(F(1,37)=20.15, p<.001, \eta_{p}{ }^{2}=.35\right)$. As reference persons did when rating themselves, players rated reference persons' expressions as significantly more anxious in the expression condition $(M=4.33, S D=1.79)$ than in the suppression condition $(M=2.33, S D$ $\left.=1.07, F(1,37)=18.15, p<.001, \eta_{p}{ }^{2}=.33\right)$, but there was no significant effect of the expression manipulation on reference persons' ratings of players' expressions $(F(1,37)=1.32$, $\left.p=.258, \eta_{p}{ }^{2}=.03\right)$. These results confirm the effectiveness of the expression manipulation using a measure based on observer ratings rather than self-report.

To check that the dispositional expressivity measure related to reference person's ongoing facial behavior during the BART procedure, we computed correlations between their BEQ scores and players' judgements of co-participants' expressed anxiety. Reference persons' BEQ scores were significantly and positively correlated with the extent to which players judged reference persons to appear more anxious than they did themselves across both framing conditions (gain: $r(40)=.33, p=.037$; loss: $r(40)=.35, p=.027$ ), and with players' ratings of the overall level of reference person's anxiety expression in the loss condition $(r(39)=.35, p=$ .027), although the corresponding correlation was not significant in the gain condition. These findings generally confirm the validity of BEQ. Reference person BEQ showed no significant correlations either with players' self-ratings of expressed or experienced anxiety or with reference persons' judgements of players' anxiety. 


\section{BART scores}

Because of our adjustment to the maximum number of balloon pumps before an explosion, we expected more trials to end in the balloon bursting than in the original version of BART. This meant that the usual measure of risk behavior based on mean number of pumps on trials that did not end in the balloon bursting (corrected BART) was based on a relatively smaller sample of the data. We therefore supplemented corrected BART score with two other measures: the first was the mean number of pumps on trials that ended in the balloon bursting (burst BART), and the second was the mean number of pumps across all trials (uncorrected BART). All three of these scores were subjected to 2 X 2 ANOVA using reward frame and expression condition as independent variables.

We found two consistent significant effects across all three dependent measures. First, there was a significant main effect of reward frame (corrected BART: $F(1,38)=4.54, p=.040$, $\eta_{p}{ }^{2}=.11$; burst BART: $F(1,38)=6.75, p=.013, \eta_{p}{ }^{2}=.15$; uncorrected BART: $F(1,38)=$ $6.12, p=.018, \eta_{p}{ }^{2}=.14$ ), showing unexpectedly that risk scores were higher in the gain version of the task than in the loss version of the task. Second there was a significant two-way interaction between reward frame and expression condition (corrected BART: $F(1,38)=5.16, p$ $=.029, \eta_{p}{ }^{2}=.12$; burst BART: $F(1,38)=4.86, p=.034, \eta_{p}{ }^{2}=.11$; uncorrected BART: $F(1$, $\left.38)=6.36, p=.016, \eta_{p}{ }^{2}=.14\right)$. Analysis of simple main effects revealed that two of the risk scores were significantly lower in the expression condition than the suppression condition for the gain task (burst BART: $F(1,38)=4.95, p=.032, \eta_{p}{ }^{2}=.12$; uncorrected BART: $F(1,38)=$ 4.54, $p=.040, \eta_{p}{ }^{2}=.11$ ), and the third showed a near-significant effect in the same direction $\left(\right.$ corrected BART: $\left.F(1,38)=3.57, p=.067, \eta_{p}{ }^{2}=.09\right)$. None of the corresponding effects were significant for the loss task (all $F \mathrm{~s}<1$, see Figure 3 for corrected BART scores). 
Simple main effects of frame on all three measures of risk behavior were strongly significant in the suppression condition (corrected BART: $F(1,38)=9.70, p=.003, \eta_{p}{ }^{2}=.20$; burst BART: $F(1,38)=11.53, p=.002, \eta_{p}^{2}=.23$; uncorrected BART: $F(1,38)=12.49, p=$ $.001, \eta_{p}{ }^{2}=.25$ ) but practically non-existent (all $\left.F_{\mathrm{s}}<.10, \eta_{p}{ }^{2}=.000\right)$ in the expression condition. No other effects were significant for any of these dependent variables ${ }^{1}$.

\section{Anxiety and appraisal ratings}

A similar significant two-way interaction between frame and expression condition affected players' ratings of experienced anxiety $\left(F(1,38)=5.25, p=.028, \eta_{p}{ }^{2}=.12\right)$. During the gain version of BART, players rated their anxiety as higher in the expression condition than in the suppression condition, whereas the reverse pattern was found for the loss version (see Figure 4). However, neither of the corresponding simple main effects was significant $\left(F_{\mathrm{s}}<1\right)$.

Expression condition had a significant main effect on players' perceptions of reference persons' appraisals of risk $\left(F(1,38)=11.92, p=.001, \eta_{p}{ }^{2}=.25\right)$, showing that players believed that reference persons thought they were taking greater risks in the anxiety-expression condition $(M=4.93, S D=1.10)$ than in the anxiety-suppression condition $(M=3.64, S D=1.29)^{2}$.

However, controlling for this effect did not remove the significant simple main effect on risk scores under the gain frame. Expression condition did not have a corresponding significant effect on players' own risk appraisals or on players' ratings of the extent to which looking at reference persons' faces made them feel more anxious.

Effects of reference person's BEQ

Reference person's BEQ showed significant negative correlations with all three risk 
measures, in both gain and loss versions of BART (all $r \mathrm{~s}<-.36$, all $p \mathrm{~s}<.025$ ), confirming that greater expressivity in reference persons led to reduced risk-taking in players. We also checked whether BEQ's effects interacted with our manipulations by adding it as a continuous independent variable to the analysis of variance of risk scores reported above. Confirming the correlational analyses, reference person's BEQ had a significant main effect on all three risk measures $\left(\right.$ corrected BART: $F(1,35)=6.85, p=.013, \eta_{p}{ }^{2}=.16$; burst BART: $F(1,35)=4.87$, $p=.012, \eta_{p}{ }^{2}=.12 ;$ uncorrected BART: $\left.F(1,35)=6.69, p=.014, \eta_{p}{ }^{2}=.16\right)$. However, BEQ was involved in no significant interactions. Because people may tend to choose friends with similar levels of expressivity to their own, it is possible that these apparent effects of reference person's BEQ actually reflected effects of correlated values of player BEQ. However, reference person's BEQ continued to have significant main effects on two out of three of our risk indices

even after controlling for players' BEQ scores (corrected BART: $F(1,32)=7.83, p=.009, \eta_{p}^{2}$ $=.20$; uncorrected BART: $\left.F(1,32)=5.98, p=.020, \eta_{p}{ }^{2}=.16\right)$.

\section{Discussion}

Social referencing and social appraisal

The primary aim of this study was to assess whether one individual's score on a performance-based measure of risk behavior could be influenced by another person's facial expression of anxiety. Our findings support such an influence in two respects. First, we successfully manipulated reference persons' facial expressions and our manipulation had significant effects on players' risk behavior on BART in the gain-frame version of this task (GBART). This finding corroborates earlier evidence collected in less controlled settings (e.g., Parkinson \& Simons, 2009), and permits firmer conclusions about the causal role of the other 
person's anxiety expression in the process. Further, by controlling interpersonal access, the present study was able to establish that the interpersonal effects of anxiety specifically depended on facial rather than verbal communication.

Second, reference persons' dispositional expressivity (as measured by BEQ) was significantly related to players' perceptions of reference persons' expressed anxiety during the task, and was a significant negative predictor of risk behavior across both versions of BART, independent of the expression manipulation. This provides the first clear evidence that individual differences in expressivity predict the extent of interpersonal influence on performance in a consequential task. Manipulation checks confirmed that more expressive reference persons gave stronger visible indications of anxiety. It therefore seems likely that their clearer and more differentiated facial signals concerning potentially threatening aspects of the task discouraged players from risk-taking.

Our reasons for predicting these effects were informed by theories of social appraisal (Manstead \& Fischer, 2001) and social referencing, and by previous findings obtained by Parkinson and Simons (2009). In particular, we expected that players would factor in their perceptions of reference persons' feelings about the task when appraising levels of risk under conditions of uncertainty. Thus, when the reference person seemed more anxious, players should have concluded that continuing to inflate the balloon was more risky than they would otherwise have thought. However, our expression manipulation did not produce significant differences in players' risk appraisals or experienced anxiety that corresponded to the significant simple main effects on indices of risk behavior that we found in the gain-frame condition, making mediation of the latter effects by the former ones seem unlikely.

One possible reason for discrepancies between effects on risk behavior and effects on 
players' risk appraisal and experienced anxiety arises from difficulties in measuring the latter mediators at a consistent level of risk across conditions. Any reductions in players' risk appraisals and experienced anxiety induced by social referencing are likely to lead to increased risk behavior, causing reciprocal increases on these mediators. For example, our findings (as presented in Figure 4) show that players' experienced anxiety was non-significantly lower in the gain-frame/suppression condition than in the gain-frame/expression condition despite the significantly higher BART scores (greater risk-taking) in the former condition, which would ordinarily lead to higher rather than lower anxiety. If anxiety had been measured at a constant level of risk across conditions (e.g. after a fixed number of pumps on a specified trial rather than at its final pump), this difference might have been significant. Similarly, if players maintained a consistent threshold of risk appraisal for deciding when to stop pumping, no significant differences in risk appraisal scores across conditions would be expected even if appraisal changes (prior to the final pump) had actually mediated the effects. Thus, the obtained effects of reference persons' anxiety expressions on players' risk behavior may still have depended partly on players' risk appraisals and experienced anxiety despite the non-significant effects on our measures of these variables. Future studies therefore need to deploy better calibrated measures. Players may also have moderated their risk-taking behavior because they believed that their partner, who also had a stake in the outcome of the task, would prefer them not to proceed (rather than because they personally felt that further pumps would be risky). In other words, the partner's apparent anxiety may have affected judgements of costs for the other rather than (or in addition to) the self, and empathy may have contributed to discontinuation of pumping. The significant effect of the expression manipulation on players' perceptions of how risky the reference person felt they were being (but not on players' own risk appraisals) is consistent with 
such an account. However, significant effects of the expression manipulation on risk scores in the gain version of the task still remained after controlling for this potential mediator. Thus it seems possible that a range of convergent social-appraisal processes operated in this study (Parkinson, 2010). Some of these processes may have operated at an implicit rather than explicit level (see Parkinson, 2011), making it difficult to capture them using self-report measures such as those used here.

\section{Framing}

Although anxiety suppression by reference persons increased players' risk-taking as predicted, our expectation that this effect would be stronger under a loss frame proved incorrect. In fact, the expression manipulation only had significant effects in the gain-frame version of BART. A likely explanation is that the loss frame already alerted participants to the threatening aspects of the task rendering them less ambiguous and therefore less open to reappraisal as a function of the reference person's communicated anxiety. Under the gain frame, threatening aspects may have become more salient only when the reference person's anxiety expression drew attention to them. Previous social referencing research in developmental psychology also demonstrates stronger effects when referenced objects are more ambiguous (e.g., Gunnar \& Stone, 1984).

The above account faces the problem that attunement to potential costs under a loss frame was predicted to increase risk-taking whereas attunement to potential costs as a result of reference persons' expressed anxiety was predicted to reduce risk-taking (and actually did reduce risk-taking under a gain frame). In this sense, our original predictions based on previous framing research could be seen as working against our predictions based on social-referencing processes. 
However, the effects of our framing manipulation did not in fact confirm predictions based on the previous findings of Benjamin and Robbins (2007), and revealed findings more consistent with our social referencing predictions. In the anxiety-suppression condition, the loss frame produced significantly lower (rather than higher) BART scores than the gain frame. In the anxiety-expression condition, frame had no impact on BART scores. Another study (Gabriel \& Williamson, 2010) published after we conducted ours also failed to replicate Benjamin and Robbins' (2007) framing effect using a modified version of BART, showing null or reverse effects across conditions as in the present study. It therefore seems that procedural variations can moderate the pattern of findings when BART methodology is used.

More generally, Levin and colleagues (1998) argue that framing manipulations directed at binary decision options between risk and safety (goal framing) as used in the present study (and in previous research into social referencing) produce less consistent effects than the framing of more complex evaluations of relative costs and benefits of independent cost-benefit trade-off alternatives (risky choice framing) as used in Kahneman and Tversky's (1979) original research (see also Levin, Gaeth, Schreiber, \& Lauriola, 2002). Goal framing implies that the correlation between outcome probability on the one hand and potential gains and losses on the other remains constant across alternative decision options, and the direction of effect may therefore depend to a greater extent on whether participants are oriented to the former or the latter (see also footnote 1, above). A number of task-related factors might potentially influence participants' orientations, thus explaining variations in results across studies.

The most obvious differences between our study and Benjamin and Robbins' earlier study arise from the interpersonal nature of our modified procedure. Our participants completed the task in pairs, had continuous access to co-participants' facial expressions, and shared the 
rewards. Although Benjamin and Robbins (2007) found no difference in framing effects when BART gains and losses were accrued by players' best friends rather than themselves, these friends were imagined and the rewards hypothetical, making their interpersonal manipulation less direct than ours. As suggested above, players completing our modified task may have adjusted their behavior in response to their co-participants' perceived risk preferences rather than basing them entirely on their own preferences (cf. Krishnamurthy \& Kumar, 2002). Further, the changing interpretational frame provided by the reference person's ongoing facial reactions to the task may have over-ridden any framing effects that might have operated had the player been individually oriented to task. In other words, the apparent reverse framing effect may in fact have simply reflected stronger influence of reference person's anxiety expressions in the gainframe condition.

Another potentially relevant procedural change is that the present study used a withinparticipants frame manipulation (see also Levin et al., 2002), which may potentially have alerted players to the formal equivalence of the two versions of BART when they performed the task for the second time. Indeed, Bernstein, Chapman and Elstein (1999) found that framing effects can be removed when participants see both versions of a risk scenario before making choices. However, additional between-participants comparisons between scores on gain and loss versions of BART using only data from the first round (i.e., before participants could have made any comparison of the counterbalanced tasks) again revealed no evidence for the predicted framing effect.

A more minor procedural alteration is that our players were not shown a constant display of points gained or lost for each balloon to supplement the indication of number of pumps. We felt that including the additional points display would encourage participants to set specific 
targets anchored against a maximum of zero points lost in the loss condition but provide no comparable maximum anchor for points gained in the gain condition. It therefore seems possible that the desire to approach zero lost points for each balloon in the loss condition (rather than the loss frame per se) was what encouraged greater risk-taking in Benjamin and Robbins' (2007) study.

\section{Limitations}

The above discussion has assumed that obtained differences in BART scores across conditions reflect induced differences in risk appraisal. However, it is possible that the reduced maximum number of pumps in our revised procedure compared to previously standardized versions made the task seem less risky to players (Lejuez et al., 2002). Further, Lejuez and colleagues (2003) point out that even participants completing the standardized BART consistently make too few pumps to allow maximization of the number of points they could conceivably earn. Thus, the apparently greater risk aversion found in our anxiety expression condition might in fact reflect less successful reward maximization. If so, reference person's anxiety expressions may have reduced BART scores because they distracted players from thinking rationally about the task rather than because they made them more concerned about potential losses. Although our data confirm that players interpreted reference persons' anxiety expressions as indicating their level of risk appraisal, it remains possible that associated distraction also played a role. This possibility could be assessed in future research by adding manipulations and assessments of cognitive load.

Another limitation of the present research arises from its exclusive focus on the expression of anxiety and its failure to assess other potentially relevant emotions. Informal 
observation of co-participants during the task suggested that reference persons' expression of amusement and excitement may also have affected players' performance. Perhaps because BART was played with a friend, our players and reference persons showed frequent smiles and occasional laughter in addition to the more obviously negative expressions we had expected. Some of these smiles might themselves be interpreted as symptoms of anxiety, but many are likely to have been more playful and worry-free.

These observations raise the possibility that expressions other than anxiety expressions may have contributed to the effects of our expression manipulation. Perhaps the anxiety suppression instruction was effective partly because it gave reference persons permission to express any amusement more freely, and perhaps the communication of amusement was one of the factors leading to increased risk-taking in the gain-frame version of BART (cf. Lerner \& Keltner, 2001). In other words, reference persons' greater levels of expressed amusement in the anxiety suppression condition may have increased players' willingness to continue with risky options. Because we did not measure amusement or happiness directly, this speculation cannot be assessed directly using the present data. However, if amused or happy expressions had indeed encouraged riskier behaviour in our study, then reference persons' dispositional expressivity should have had a positive effect on BART scores rather than the negative effect we in fact obtained. Nevertheless, future research should investigate the role of other potentially riskrelevant emotion expressions in addition to anxiety. It will also be important to determine whether such effects depend on prior friendly relations between players and reference persons. It is even possible that interpersonally expressed anxiety may contribute to the excitement of the task as well as discouraging continuation under certain circumstances. 


\section{Conclusions}

The present study was the first to use BART as a dyadic rather than individual risk task, and clearly demonstrates that theoretically interesting and practically applicable findings can be generated using this extension of the original methodology. In particular, we found as predicted that manipulations of, and individual differences in, the emotional expressivity of a reference person had significant effects on players' perceptions of this reference person's expressions, own reported anxiety during the task, and performance-based objective measures of risk-taking. These findings conclusively confirm the operation of adult social referencing effects in a more controlled setting than used in our earlier research (Parkinson \& Simons, 2009). More specifically, suppressing anxiety expression seems to encourage friends to take more risky choices when tasks are framed in terms of potential gains rather than losses. Such a finding has obvious implications for the interpersonal emotion regulation of advisors or counsellors intervening in real-world decision-making situations. We believe that the methodology developed here has great potential for generating further theoretically significant and practically applicable findings concerning interpersonal effects of emotion expression. 


\section{References}

Aklin, W. M., Lejuez, C. W., Zvolensky, M. J., Kahler, C. W., \& Gwadz, M. (2005). Evaluation of behavioral measures of risk taking propensity with inner city adolescents. Behaviour Research and Therapy, 43, 215-228.

Benjamin, A., \& Robbins, S. (2007). The role of framing effects in performance on the Balloon Analogue Risk Task (BART). Personality and Individual Differences, 43, 221-230.

Bernstein, L. M., Chapman, G. B., \& Elstein, A. S. (1999). Framing effects in choices between multi-outcome life-expectancy lotteries. Medical Decision Making, 19, 324-338.

Bornolova, M. A., Cashman-Rolls, A., O’Donnell, J. M., Ettinger, K., Richards, J. B., de Wit, H., \& Lejuez, C. W. (2009). Risk taking differences on a behavioral task as a function of potential reward/loss magnitude and individual differences in impulsivity and sensation seeking. Pharmacology, Biochemistry and Behavior, 93, 258-262

Brewer, M. B., \& Kramer, R. M. (1986). Choice behavior in social dilemmas: Effects of social identity, group size, and decision framing. Journal of Personality and Social Psychology, $50,543-549$.

Butler, E. A., Egloff, B., Wilhelm, F. H., Smith, N. C., Erickson, E. A., \& Gross, J. J. (2003). The social consequences of expressive suppression. Emotion, 3, 48-67.

Butler, E. A., \& Gross, J. J. (2004). Hiding feelings in social contexts: Out of sight is not out of mind. In P. Philippot \& R. S. Feldman (Eds.), The regulation of emotion (pp. 101-126). Mahwah, NJ: Erlbaum.

Crowley, T. J., Raymond, K. M., Mikulich-Gilbertson, S. K., Thompson, L. T., \& Lejuez, C. W. (2006). Risk-taking "set" in a novel task among adolescents with serious conduct and substance problems. Journal of the American Academy of Child and Adolescent 
Psychiatry, 45, 175-183.

Ekman, P., \& Friesen, W. V. (1969). Nonverbal leakage and clues to deception. Psychiatry: Journal for the Study of Interpersonal Processes, 32, 88-106.

Fagley, N. S., Coleman, J. G., \& Simon, A. F. (2010). Effects of framing, perspective taking, and perspective (affective focus) on choice. Personality and Individual Differences, 48, 264269.

Gabriel, K. I., \& Williamson, A. (2010). Framing alters risk-taking behaviour on a modified Balloon Analogue Risk Task (BART) in a sex-specific manner. Psychological Reports, 107, 699-712.

Gross, J. J. (1998). Antecedent- and response-focused emotion regulation: Divergent consequences for experience, expression, and physiology. Journal of Personality and Social Psychology, 74, 224-237.

Gross, J.J., \& John, O.P. (1995). Facets of emotional expressivity: Three self-report factors and their correlates. Personality and Individual Differences, 19, 555-568.

Gunnar, M. R., \& Stone, C. (1984). The effects of positive maternal affect on infant responses to pleasant, ambiguous, and fear-provoking toys. Child Development, 55, 1231-1236.

Harrison, J. D., Young, J. M., Butow, P., Salkeld, G., \& Solomon, M. J. (2005). Is it worth the risk? A systematic review of instruments that measure risk propensity for use in the health setting. Social Science and Medicine, 60, 1385-1396.

Heilman, R. M., Crisan, L. G., Houser, D., Miclea, M., \& Miu, A. C. (2010). Emotion regulation and decision making under risk and uncertainty. Emotion, 10, 257-265.

Holodynski, M., \& Friedlmeier, W. (2006). Development of emotions and emotion regulation. New York: Springer. 
Hsee, C. K., \& Weber, E. U. (1997). A fundamental prediction error: Self-others discrepancies in risk preference. Journal of Experimental Psychology: General, 126, 45-53.

Kahneman, D., \& Tversky, A. (1979). Prospect theory: An analysis of decisions under risk. Econometrica, 47, 313-327.

Krishnamurthy, P., \& Kumar, P. (2002). Self-other discrepancies in waiting time decisions. Organizational Behavior and Human Decision Processes, 87, 207-226.

Lanzetta, J. T., Cartwright-Smith, J., \& Kleck, R. E. (1976). Effects of nonverbal dissimulation on emotional experience and autonomic arousal. Journal of Personality and Social Psychology, 33, 354-370.

Latané, B., \& Darley, J. M. (1968). Group inhibition of bystander intervention in emergencies. Journal of Personality and Social Psychology, 10, 215-221.

Lejuez, C. W., Read, J. P., Kahler, C. W., Richards, J. B., Ramsey, S. E., Stuart, G. L., et al. (2002). Evaluation of a behavioral measure of risk taking: The Balloon Analogue Risk Task (BART). Journal of Experimental Psychology: Applied, 8, 75-84.

Lerner, J. S., \& Keltner, D. (2001). Fear, anger, and risk. Journal of Personality and Social Psychology, 81, 146-159.

Levin, I. P., Schneider, S. L., \& Gaeth, G. J. (1998). All frames are not created equal: A typology and critical analysis of framing effects. Organizational Behavior and Human Decision Processes, 76, 149-188.

Levin, I. P., Gaeth, G. J., Schreiber, J., \& Lauriola, M. (2002). A new look at framing effects: Distribution of effect sizes, individual differences, and independence of types of effects. Organizational Behavior and Human Decision Processes, 88, 411-429.

Loewenstein, G. F., Weber, E. U., Hsee, C. K., \& Welch, N. (2001). Risk as feelings. 
Psychological Bulletin, 127, 267-286.

Manstead, A. S. R., \& Fischer, A. H. (2001). Social appraisal: The social world as object of and influence on appraisal processes. In K. R. Scherer, A. Schorr, \& T. Johnston (Eds.), Appraisal processes in emotion: Theory, methods, research (pp. 221-232). New York: Oxford University Press.

Parkinson, B. (1996). Emotions are social. British Journal of Psychology, 87, 663-683.

Parkinson, B. (2010). Interpersonal emotions. In K. R. Scherer et al. (Eds), Blueprint for an affectively competent agent: A sourcebook (pp. 131-147). Oxford: Oxford University Press.

Parkinson, B. (2011). Interpersonal emotion transfer: Contagion and social appraisal. Personality and Social Psychology Compass, 5,

Parkinson, B., \& Simons, G. (2009): Affecting others: Social appraisal and emotion contagion in everyday decision making. Personality and Social Psychology Bulletin, 35, 1071-84.

Rao, H., Korczykowski, M., Pluta, J., Hoang, A., \& Detre, J. A. (2008). Neural correlates of voluntary and involuntary risk taking in the human brain: An fMRI study of the balloon analog risk task (BART). Neuroimage, 42, 902-910.

Sorce, J. F., Emde, R. N., Campos, J., \& Klinnert, M. D. (1985). Maternal emotional signaling: Its effect on the visual cliff behavior of 1 year olds. Developmental Psychology, 21, 195200.

Van Kleef, G. A. (2009). How emotions regulate social life: The emotions as social information (EASI) model. Current Directions in Psychological Science, 18, 184-188. 


\section{Author Note}

This research was supported by the Economic and Social Research Council (UK) as part of project EROS (Emotion Regulation of Others and Self, RES-060-25-0044). We are also grateful to Irene Rodriguez for assistance in running participants, to Simon Lutterbie for help with programming of the BART tasks, and to Carl Lejuez, Michael Crowley, Andreu Vigil-Colet, and Steven Robbins for sharing information about BART and their adjustments to it. 


\section{Footnotes}

1. Following recently published research by Gabriel and Williamson (2010), we checked the extent to which the effects of frame and expression on risk scores were moderated by participant gender. Gender had a significant main effect on only one of our risk measures $\left(\right.$ corrected BART: $\left.F(1,36)=4.39, p=.043, \eta_{p}{ }^{2}=.11\right)$, showing lower risk scores for women than for men, and there were no significant interactions between gender and other independent variables for any of the three risk measures.

2. One reviewer of a previous version of this manuscript suggested that players may have believed that reference persons' anxiety was about missing out on possible points because of them making too few pumps rather than about the balloon exploding. The finding reported here effectively rules out this interpretation. As expected, players judged that reference persons in the anxiety-expression condition attributed higher and not lower risk-levels to their (i.e., players') behaviour. 


\section{Figure Captions}

Figures $1 \mathrm{a}$ and $1 \mathrm{~b}$. Procedures and displays for gain-frame and loss-frame versions of BART.

Figure 2. Co-participants' facial expressions in suppression and expression conditions (reference person in cubicle $\mathrm{C}$ shown on the right of each picture).

Figure 3. Interactive effects of reward frame and expression condition on corrected BART scores.

Figure 4. Interactive effects of reward frame and expression condition on players' experienced anxiety. 


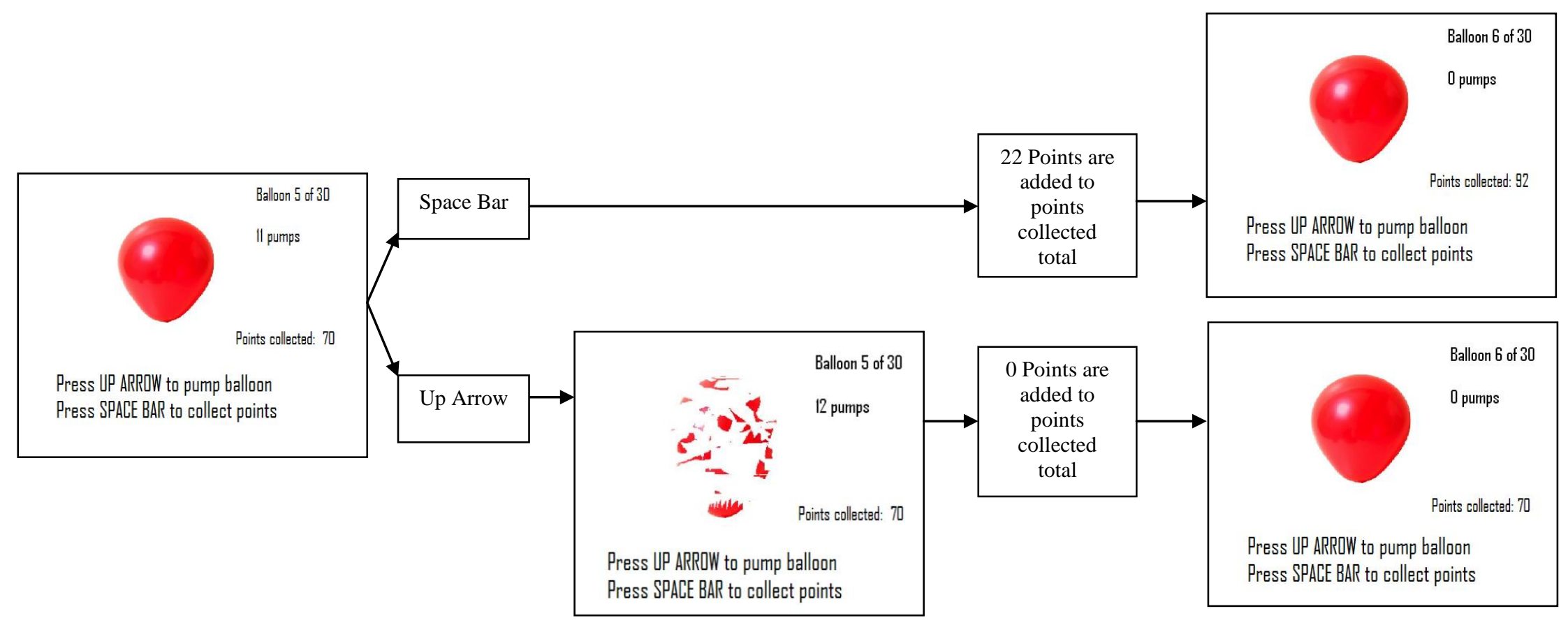

Figure 1a: Gain task. "Points collected" indicates the number of points already gained during the game. If the player presses the space bar, 22 points are banked (earned from 11 pumps without bursting the balloon), bringing the total of points collected up to 92 . If the player pumps again by pressing the up arrow, the balloon bursts and no points are added, leaving the total of collected points at 70 . 


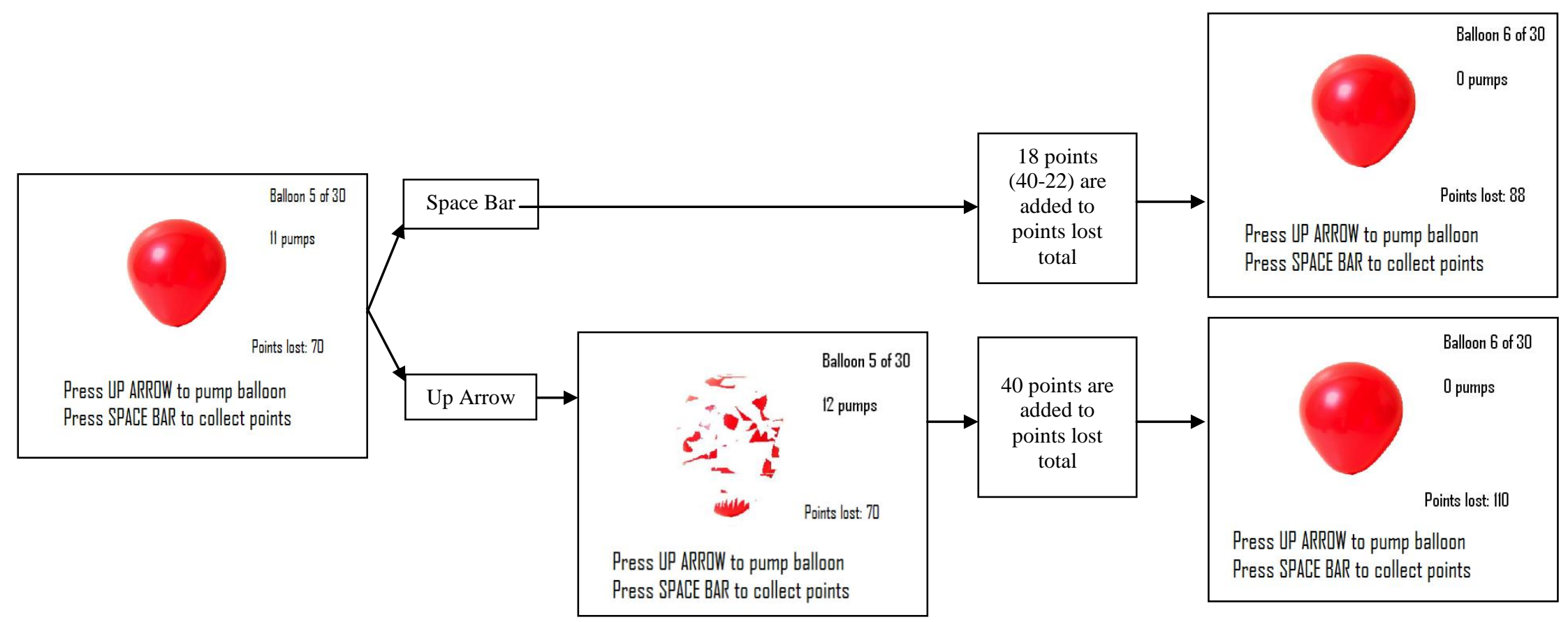

Figure 1b: Loss task. "Points lost" indicates the number of points already lost during the game. If the player presses the space bar, 22 points are saved (earned from 11 pumps without the balloon bursting), adding 18 points (the balloon's maximum value of 40 minus 22 saved points) points to the total of lost points (now 88). If the player presses the up arrow, the balloon bursts and the player loses the balloon's maximum value of 40 points, bringing the total of lost points up to 110 . 

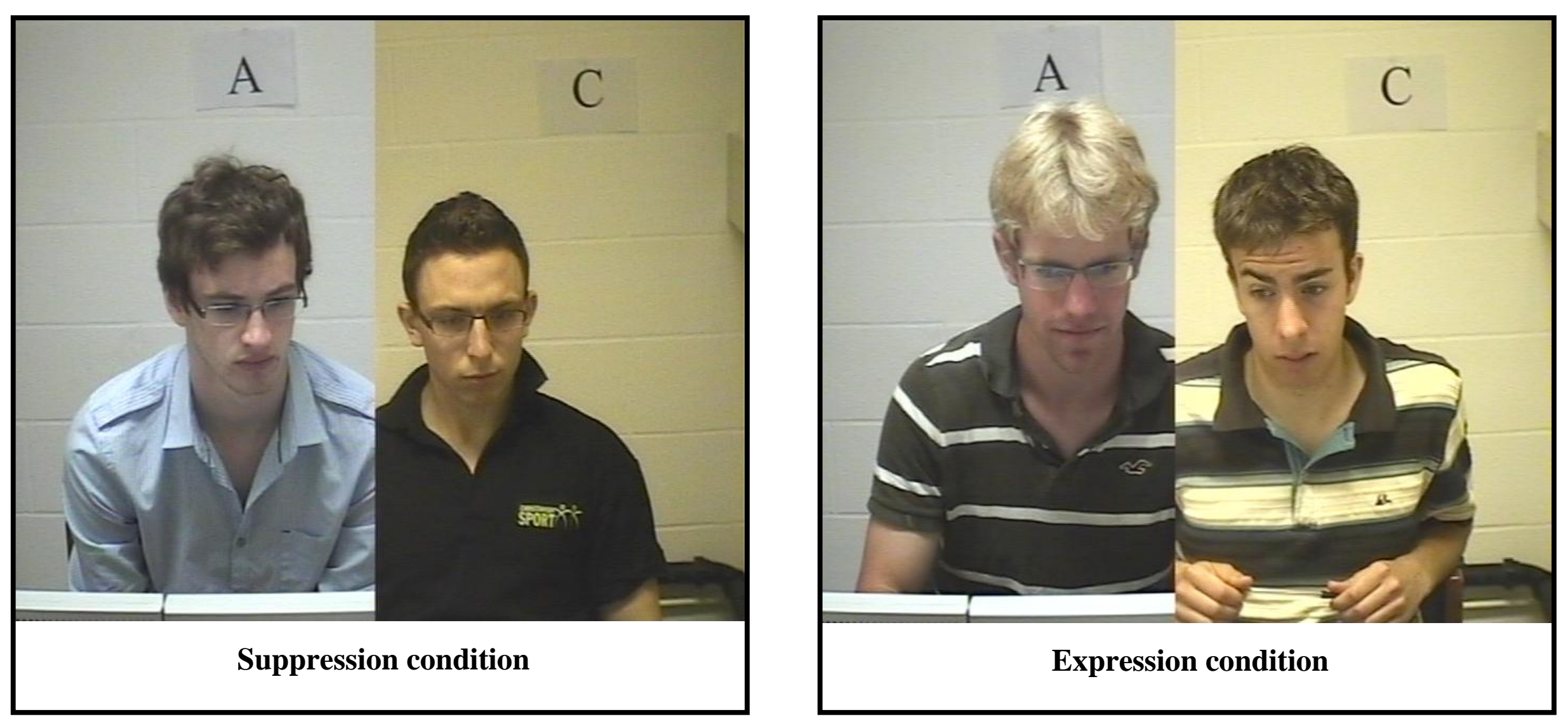


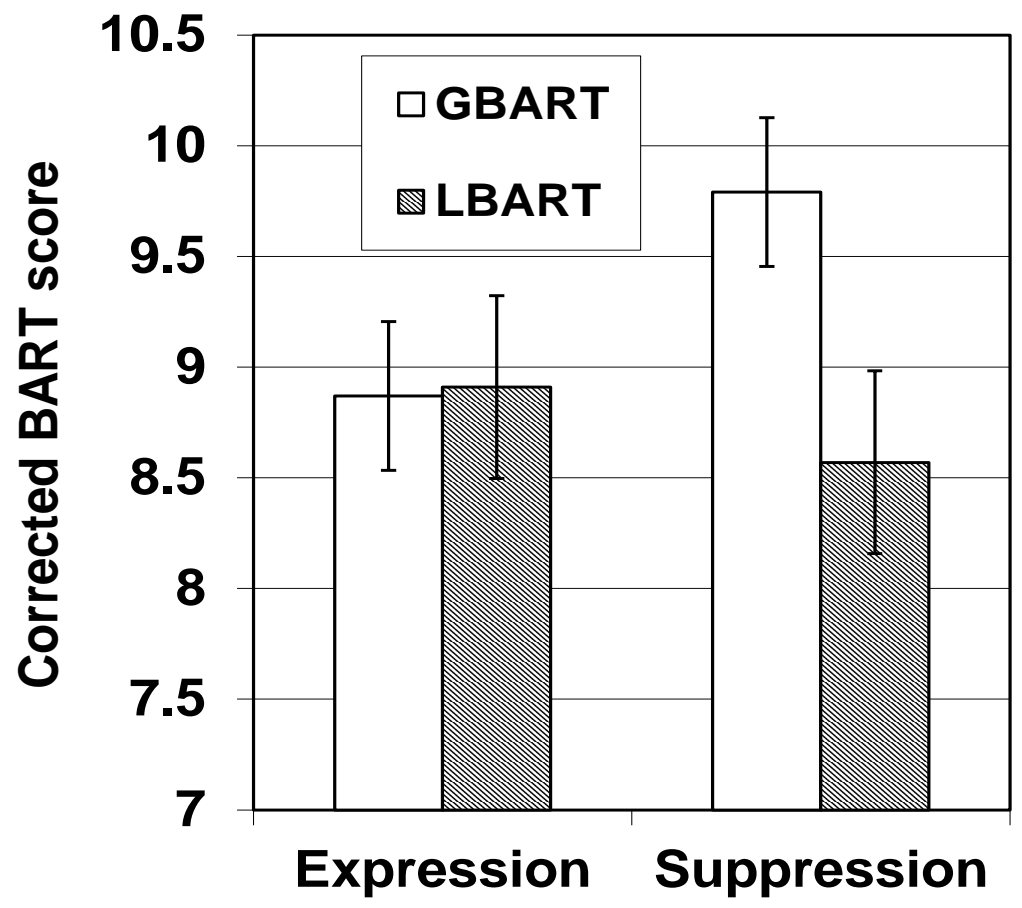




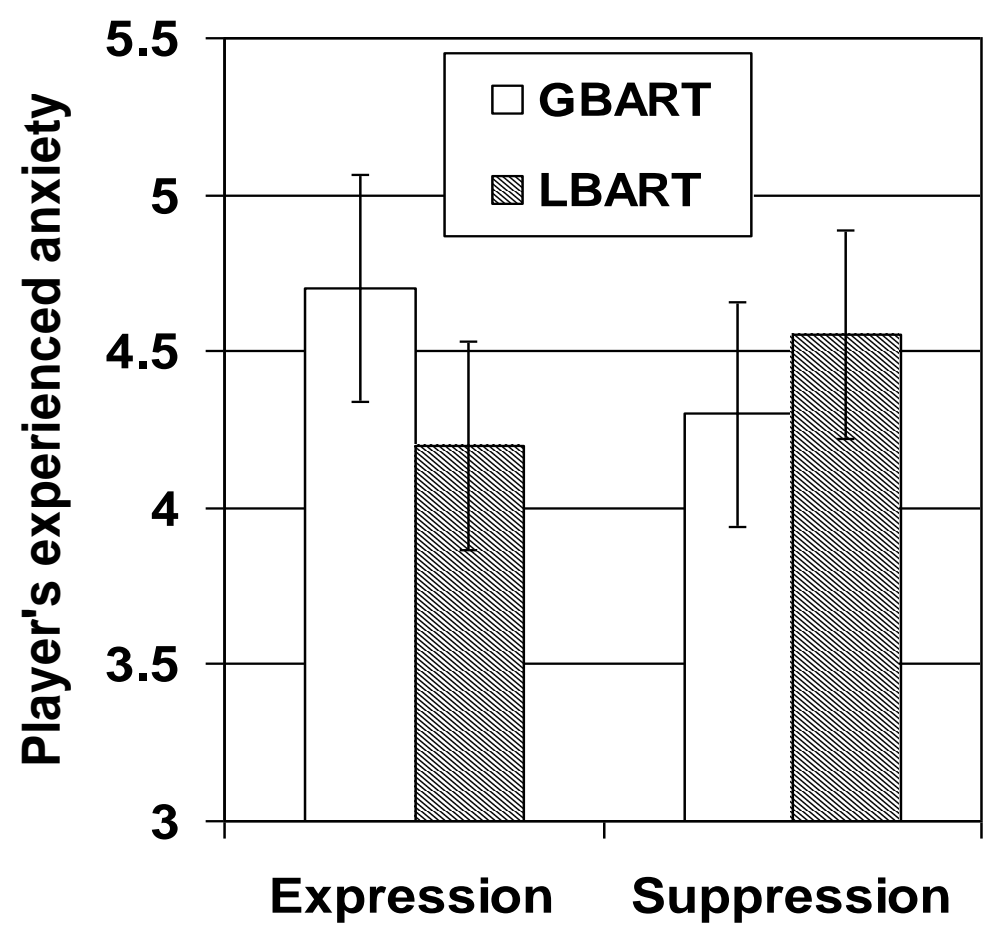

